\title{
Charm of Splicing in Fashion
}

\author{
Xuemei Xu \\ Jiang xi Institute of Fashion Technology \\ Nanchang, Jiang xi, China 330201
}

\begin{abstract}
The de velopment of modern politics and economy drive the continuous upgrade of the fashion industry. In modern fashion field, mastering leadingedge fashion information is possible to make a success. As fashion designers, we have many techniques to use in fashion design. Splicing is the most commonly used design technique, and is widely used in fashion design. Starting from the form beauty of fashion design, the author has researched all kinds of splicing methods, including structure splicing, color splicing, fabric splicing and style splicing, expounded the design charm and advantages of each splicing method, the important effect of splicing on the whole fashion style. It is the basis of outer contour of fashion. We cannot work behind closed door in fashion design. Based on the market environment, the paper has combined market environment with fashion splicing technique in order to support the splicing fashion.
\end{abstract}

Keywords—splicing; fashion design; fashion modeling; market

\section{INTRODUCTION}

In the information age, society, politics and economy develop rapidly. Fashion faces the trend of upgrade and development again and again in large social environment. The direction of modern diversified fashion is clearer, and fashion design has get rid of outdated design thinking and entered into the multidimensional design field. At the same time, the trend of intelligent fashion design is becoming more and more popular at home and abroad. Some research institutions also have the corresponding scientific achievements. Although they are not immature, the intelligent development direction is an unstoppable trend.

In the background of international and domestic intelligent development, the fashion industry faces a higher upgrading trend. Under the influence of high upgrade, the author starts from design techniques, takes relatively classic splicing technique as entry point and focuses on the splicing technologies and methods. It is to guide fashion design to develop to multi-angle design thinking, get rid of single structure splicing, introduce color splicing, link splicing color match with popular colors to highlight the importance of popular colors. We can introduce fabric splicing, achieve seamless stitching of thin and thick and functional differences to form a complete match mode, and fully affirm modern new fabrics. For pattern splicing, we should pay more attention to the imaginary beauty between gather and dispersing, so as to give consumers unlimited imagination. The author mainly researches fashion splicing design and asymmetric techniques. The paper has analyzed fashion splicing technique from all aspects. It, with real objects on fashion stage as example, has analyzed its design advantages from multi-angle in order to provide reference for subsequence splicing technique research of designers or fashion enterprises.

\section{STRUCTURE SPLICING}

Structure splicing is a basic splicing method in fashion design. It is to stitch and splice basic structures of fashion, and to ensure the three-dimensional effect of fashion. Generally structure splicing is very simple. Stitch structure cut-parts together according to the making requirements and form a three-dimensional modeling to enclose body. It is a basic splicing technique in fashion modeling design. With structure splicing as design basis, most structure splicing parts are in side seam, back center, collar and etc. For other non-structure splicing, on the bas is of it, segment the clothes and then resplice them together.

The requirements for modern fashion modeling is becoming increasingly high, as well as for splicing techniques. The author has engaged in fashion structure design for many years. It is advisable to use complete stitching splicing or nonstitching overlap splicing for collar and clothes body. We can use adhesive buckles, buttons or buttonholes to achieve the stitching effect. We can use detachable collar to increase function of the clothes and bring new design mode and promote the development of fashion industry. Fashion design style shall closely follow the popular trend. An alternative stitching splicing appears in the market. The characteristic is that the lengths of the front and the back are different. The side seam stitching makes the back with a longer piece. The longer piece has formed independent back body piece after wrapping technology. This style of splicing is derived from the modeling of swallow-tailed coat. It has become fashionable marketable goods once it was put on international popular stage.

\section{COLOR SPLICING}

Today, people's material and spiritual life are great satisfied with the improvement of living standard. People begin to pursue high-quality enjoyment in spirit. People's color sensitivity is much higher than their shape sensitivity. Their pursuit for clothes is not simply involved in packing body. Their requirements for design, style and color are becoming higher and higher. The requirements for the first impression color shall be higher. Due to different structure, modeling and fabrics, fashion colors certainly bring different color splicing. In fashion design, the splicing colors should be not overmuch in order to keep the personality of clothes. Multi-color splicing fashion is garish, leaving people a non-steady and immature 
sense. Fashion with perfect color match will not keep people's attention on fashion itself, because the whole beauty of fashion concerning human body is the purpose of fashion design.

In splicing fashion, there are the following color match methods: first is the splicing of similar colors. Due to same color and hue quality, the only difference is on shade of color in the splicing. The color gap is relatively small. The difference in lightness and purity can bring a sense of rhythm and rhyme. It is a stable and steady color splicing method. Second is the contrast color splicing. The color difference is relative large. There is a strong contrast effect in color, lightness, purity and changes in temperature. It is a very popular color match for young people. The splicing methods are various, and they can produce strong visual impact to stimulate the vitality and youth of young people. The last is the complementary color splicing. These colors are spliced together due their complementary function. Each color has its own advantage. They make the best of all colors, mutually cooperate and jointly promote the overall sense of fashion. Color collision always adds people's life with fun. In recent years, the collision color splicing is a popular theme. The collision color refers to contrast color or complementary color, with large color difference, colliding out youth and vitality. The publicity personality is the hot pursuit of modern young people.

\section{FABRIC SPLICING}

As one of the three elements of clothing, fabric has played a key role in the three-dimensional modeling. The original splicing art is originated from fabric splicing. It was in the era lacking of materials, so leftover materials were reused. This splicing method was to save materials at that time. In modern time, we also oppose extravagance and waste, so splicing fashion once again becomes an aesthetic trend of fashion. In modern times, a variety of fabrics of different thickness, functions, athletics and colors are full of market. Due to different features, the splicing of different fabrics will produce different splicing effect. The splicing art of fashion itself is a little difficult. In researching fabric splicing, the author has summarizes some matters needing attention. Only by solving the splicing difficulty of fabrics, can the whole splicing effect of fashion be perfectly presented.

There are several fabric splicing modes. First is the splicing of same fabric. With same thickness, function and ironing temperature, it can reduce the complication of the production. We only need to pay attention to color difference of splicing fabric. In recent years, the fabric splicing with different thickness is very popular, especially in winter clothes. Designers add lace fabric and organza fabric with light weight, and splice with wool fabric or feather fabric by the use of the splicing and collage method. The thickness, warmth, ironing temperature and color fading of the fabrics are very different, so the stitching and ironing temperature shall be taken into consideration in splicing. The light and thin fabric mainly is to adjust the overall effect and add interestingness of the clothing. For special material, the difficulty mainly lies on process design and subsequent washing. Recently, the PVC transparent plastic material is very popular. Splice it with soft fabric, producing an elegant and luxury effect. We use high-tech materials in fashion in order to get a good comprehensive effect. But the later washing and maintenance are troubles.

\section{PATTERN SPLICING}

In fashion design, the fabric color without pattern is the visual impact. For fabrics with pattern, the pattern can add visual focus to clothes. Through the ages, the development and design of pattern emerge in endlessly. Fashion pattern design can get rid of the empty feeling of single color fabric, and give fashion with freshly new visual impact. The fashion pattern splicing the author researched is established on the basis of structure splicing. There are direct and indirect methods in structure and pattern splicing technique. The pattern design of front fly generally uses indirect overlap splicing. The patterns show a dynamic sense with the movement of human body. It can give fashion pattern a dynamic sense and increase the flexibility of fashion and stimulate the vitality of wearers. Another is the split-type indirect splicing, slitting from the lower half part of the side seam or the front and back of the skirt. This indirect pattern splicing gives people a passive sense of splicing. But it can increase consumer attention on fashion. At the same time, with the separation and reunion of the fabrics, patterns give human body a faint beauty. The association and imagination can increase the added value of clothing.

Orderly pattern splicing mainly takes geometric modeling as primary. The regular and organized splicing can increase the dependent sense and directionality of fashion, which has certain guiding effect. It is a pattern design that designers often use. The un-orderly pattern splicing is to re-arrange patterns on the basis of orderly pattern. With careful design of designer, it can bring consumers free regular design thinking. Of course, the design shall conform to the aesthetic rules and characteristics, so that consumers would love this style and they can be sold out. Regardless of pattern of splicing, as long as the design ideas are kind and natural, there will always be consumers to appreciate it.

\section{ST YLE SPLICING}

Style design occupies an important position in three elements of fashion design. On fashion stage, style and modeling elements have been refreshed many times. And then the trend of fashion style and modeling appeared. The style splicing of fashion is a secondary splicing based on the structure splicing. There are various splicing forms. According to fashion modeling and dynamic element of human body, we can get many style splicing forms. The splicing form determines the style of the fashion. The style splicing has made an undeniable contribution to increasing the individuality of fashion.

The style splicing art includes detachable style and nondetachable style. It has a strong meaning of decoration. The detachable splicing is to separate or split fashion into multiple parts. The splicing part can be shoulder line. The clothes without sleeves become a vest. The effect of multi-purpose clothes appears. The splicing part can be thigh part. Install the two trouser legs and then become trousers. In addition, in this mode, the dress can become a short upper garment. If one 
piece clothes have multi-purpose, the beneficiaries are consumers. For non-detachable splicing, we can use left-right, front-back and up-down splicing to produce an asymmetry, two-sided or tall and slim effect. With various design techniques, the originality of fashion is thus greatly increased. It becomes the focus of design. The style splicing also has the flavor of multipurpose. Splice some special materials in the special parts of clothes to extend the service life. Generally, we can see functional splicing art in cuffs, hem, skirt hem, trouser legs, and front fly.

In general, splicing technique, as a fashion design expression method, is a very common design method in fashion design in recent years. And it is very popular in fashion market. It has become a new trend of fashion consumption. In addition to structure splicing, pattern splicing, style splicing, color splicing and fabric splicing, there are some other special-purposed fashion splicing, such as astronaut costumes, clothes for firefighters in the fire service, police bulletproof clothing and so on. The use of splicing enables to make full play of the beauty of fashion, shows the fashion design concepts and fashion culture, and paves a way suitable for modern aesthetic needs. The author believes that it is an urgent task to use the creative thinking of modern splicing design to design more works suitable for the public demands.

\section{REFERENCES}

[1] He Xinrong. Innovation and Application of Splicing Design in Sports Apparel. Zhejiang Sci-Tech University, Jan. 14, 2013.

[2] Yuan Bo. Analysis of Application of Splicing in Fashion Design. Popular Literature and Arts, Jul. 30, 2014.

[3] Xu Yi. Application of Splicing in Fashion Design. Beijing Institute of Fashion T echnology, Jun. 1, 2012.

[4] Lv Xinying. Beauty of Cloth Splicing Art in Fashion Design. Modern Decoration (Theory), Dec. 15, 2014.

[5] Dan Ling. Research on Application of Splicing Techniques in Sweater Design. Xi'an Polytechnic University, Dec. 9, 2012.

[6] Tu Chenchen. Research and Exploration of Cloth Splicing Art in Fashion Design. Dalian Industry University, Mar. 2011.

[7] Yu Lan. Application of Innovative Splicing Technique in Dress Design. Tianjin T extile Science and T echnology, May 15, 2014 